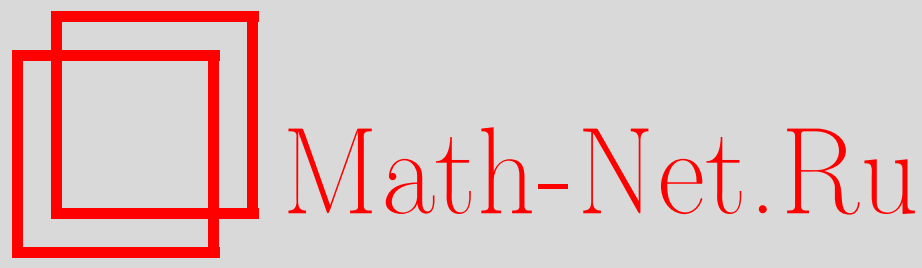

Ю. В. Чугреев, $k$-Эссенция в релятивистской теории гравитации и общей теории относительности, ТМФ, 2018, том 194, номер 3, 510-521

DOI: https://doi.org/10.4213/tmf9347

Использование Общероссийского математического портала Math-Net.Ru подразумевает, что вы прочитали и согласны с пользовательским соглашением http://www.mathnet.ru/rus/agreement

Параметры загрузки:

IP: 52.23 .180 .231

26 апреля 2023 г., 16:21:02

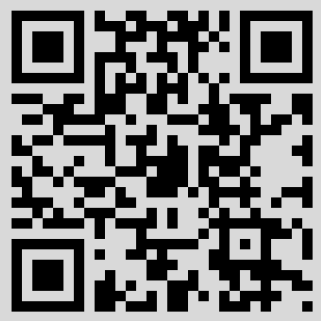




\title{
ФИЗИКА
}

Том 194, № 3

март, 2018

(C) 2018 г.

Ю. В. Чугреев

\section{$k$-ЭССЕНЦИЯ В РЕЛЯТИВИСТСКОЙ ТЕОРИИ ГРАВИТАЦИИ И ОБЩЕЙ ТЕОРИИ ОТНОСИТЕЛЬНОСТИ}

\begin{abstract}
Рассмотрена модель скалярного поля с нетривиальной кинетической частью $(k$-эссенции) на фоне плоской однородной и изотропной Вселенной в рамках релятивистской теории гравитации и ОТО. Такое скалярное поле имитирует вещество идеальной жидкости и служит моделью темной энергии, поскольку на поздних временах оно приводит к космологическому ускорению. Для целей поиска адекватного космологического сценария удобнее задавать зависимость плотности энергии такого поля от масштабного фактора, а уже потом находить соответствующий ему лагранжиан. На основе решения такой обратной задачи показано, что в релятивистской теории гравитации любое скалярное поле этого типа либо приводит к нестабильностям, либо стадия сжатия заканчивается недопустимо рано. Отмечается, что непротиворечивой моделью темной энергии в релятивистской теории гравитации может стать скалярное поле с отрицательным потенциалом (экпирозис) Стейнхардта-Турока. В ОТО модель $k$-эссенции жизнеспособна и может представлять собой как темную энергию, так и темную материю. Рассмотрен ряд конкретных моделей $k$-эссенции.
\end{abstract}

Ключевые слова: РТГ, $k$-эссенция, обратная задача, экпирозис, газ Чаплыгина.

DOI: https://doi.org/10.4213/tmf9347

\section{1. ВВЕДЕНИЕ}

Тридцать лет назад в работе [1] был предложен осциллирующий сценарий развития фридмановской Вселенной в релятивистской теории гравитации (РТГ), в которой тензорное гравитационное поле $\phi^{\mu \nu}$ является массивным и описывается известными уравнениями

$$
\begin{aligned}
R_{\mu \nu}-\frac{m^{2}}{2}\left(g_{\mu \nu}-\gamma_{\mu \nu}\right) & =\frac{8 \pi G}{c^{4}}\left(T_{\mu \nu}-\frac{1}{2} g_{\mu \nu} T\right), \\
D_{\beta}\left(\sqrt{-g} g^{\alpha \beta}\right) & =0
\end{aligned}
$$

* Московский государственный университет им. М. В. Ломоносова, Москва, Россия. E-mail: chugreev@physics.msu.ru 
где $m$ - масса гравитона, $\gamma_{\mu \nu}$ - метрика Минковского, в которой задана ковариантная производная $D_{\beta}, g_{\mu \nu}$ - метрика эффективного риманова пространства-времени:

$$
\sqrt{\frac{\operatorname{det} g_{\mu \nu}}{\operatorname{det} \gamma_{\mu \nu}}} g^{\alpha \beta}=\gamma^{\alpha \beta}+\phi^{\alpha \beta},
$$

$R_{\mu \nu}(g)$ - тензор Риччи, $T_{\mu \nu}$ - тензор энергии-импульса материи, $G=M_{\mathrm{Pl}}^{-2}$ - гравитационная постоянная, $c$ - скорость света. Греческие индексы пробегают значения $0,1,2,3$, а латинские - $1,2,3$. В дальнейшем мы будем использовать планковскую систему единиц $G=c=\hbar=k=1$. При $m=0$ уравнения (1), (2) формально совпадают с уравнениями ОТО в гармонических координатах. Добавление к ним членов с массой гравитона изменяет характер фридмановского однородного и изотропного решения ОТО. Для плоской Вселенной ${ }^{1)}$ с метриками

$$
\begin{aligned}
& d s^{2} \equiv g_{\alpha \beta} d x^{\alpha} d x^{\beta}=a(t)^{6} d t^{2}-\beta^{4} a^{2}(t)\left(d \mathrm{x}^{2}+d \mathrm{y}^{2}+d \mathrm{z}^{2}\right), \\
& d \sigma^{2} \equiv \gamma_{\alpha \beta} d x^{\alpha} d x^{\beta}=d t^{2}-\left(d \mathrm{x}^{2}+d \mathrm{y}^{2}+d \mathrm{z}^{2}\right)
\end{aligned}
$$

где $a(t)$ - масштабный фактор, $\beta$ - константа интегрирования уравнения (2), получаются следующие космологические уравнения:

$$
\begin{aligned}
\left(\frac{\dot{a}}{a}\right)^{2} & =\frac{8 \pi}{3} \rho-\frac{m^{2}}{12}\left[2-\frac{3}{\beta^{4} a^{2}}+\frac{1}{a^{6}}\right], \\
\frac{\ddot{a}}{a} & =-\frac{4 \pi}{3}(\rho+3 p)-\frac{m^{2}}{6}\left[1-\frac{1}{a^{6}}\right] .
\end{aligned}
$$

Как обычно, точка “." обозначает производную по собственному времени $d / d \tau$ $\left(a^{3} d / d t=d / d \tau\right), \rho(\tau), p(\tau)$ - плотность и давление вещества Вселенной соответственно.

В ОТО есть только один (риманов) интервал, который для случая плоской фридмановской Вселенной имеет вид

$$
d s^{2} \equiv g_{\alpha \beta} d x^{\alpha} d x^{\beta}=d \tau^{2}-a^{2}(t)\left(d \mathrm{x}^{2}+d \mathrm{y}^{2}+d \mathrm{z}^{2}\right)
$$

и приводит к тем же уравнениям (6), (7), если в них положить $m=0$.

Из закона сохранения энергии вещества

$$
\dot{\rho}+3(\rho+p) \frac{\dot{a}}{a}=0
$$

следует, что при простейшем выборе уравнения состояния $p=\omega \rho, \omega=$ const, плотность $\rho$ зависит от масштабного фактора так же, как в ОТО $\left(A_{\omega}=\right.$ const):

$$
\rho=\frac{A_{\omega}}{a^{3(1+\omega)}} .
$$

До открытия космологического ускорения плотность вещества (9) считалась двухкомпонентной, состоящей из вещества и излучения:

$$
\rho=\frac{A_{\mathrm{M}}}{a^{3}}+\frac{A_{\mathrm{r}}}{a^{4}}
$$

1) Вообще говоря, уравнения (1) и (2) допускают и фридмановские решения с отрицательной пространственной кривизной [2]. 
поэтому возникали две разворотные точки - при высоких плотностях $(a \ll 1)$ отрицательный член, пропорциональный $\mathrm{m}^{2} / a^{6}$, “догонял” преобладающую в этой фазе радиацию, а при достаточно больших $а$ малая плотность материи (темная материя, барионы и нейтрино) неизбежно должна была стать равной $m^{2} / 16 \pi$, где и происходил отскок $\left(a=a_{\max }\right)$ с последующим развитием коллапса Вселенной к $a_{\min }$.

Масса гравитона проявляла себя только вблизи точек разворота, в остальном фридмановский сценарий ОТО не менялся. В работе [3] было показано, что при $\beta^{4} \geqslant a_{\max }^{4}$ такое решение удовлетворяет принципу причинности РТГ, поэтому в уравнении (6) пропорциональный $a^{-2}$ член хотя и уменьшается медленнее, чем $\rho(10)$, но не может участвовать в фиксации $a_{\max }$.

После открытия на рубеже веков космологического ускорения и изменения знака параметра замедления появилась необходимость добавления к традиционному составу вещества (10) либо космологического члена (с положительным знаком), либо квинтэссенции с отрицательным давлением [4], [5]. Вариант с космологическим членом был отвергнут, так как на поздних временах он приводил к инфляционному разлету с неизбежным нарушением принципа причинности. Поэтому был избран вариант квинтэссенции с медленно меняющейся плотностью:

$$
\begin{gathered}
\rho=\frac{A_{\mathrm{M}}}{a^{3}}+\frac{A_{\mathrm{r}}}{a^{4}}+\frac{A_{\nu}}{a^{3 \nu}}, \\
p_{\nu}=-(1-\nu) \rho_{\nu}, \quad 0<\nu<\frac{2}{3} .
\end{gathered}
$$

Позднее в работе [6] был предложен лагранжиан скалярного поля $k$-эссенции с нетривиальной кинетической частью, реализующего эту модель:

$$
\begin{aligned}
L_{\phi} & =\sqrt{-g} K(X), & X & =\frac{1}{2} g^{\alpha \beta} \nabla_{\alpha} \phi \nabla_{\beta} \phi, \\
K(X) & =-\epsilon X^{-\nu / 2(1-\nu)}, & \epsilon & =\text { const }>0,
\end{aligned}
$$

где $\nabla_{\alpha}$ - ковариантная производная по метрике $g_{\mu \nu}, \phi$ - скалярное поле.

В статье [7] на основании изучения движения галактик в Местной группе было установлено, что постоянный параметр квинтэссенции $\nu$ должен быть очень мал: $0<\nu \leqslant 0.05$.

Во втором разделе настоящей статьи для скалярного поля $k$-эссенции простейшего вида (13) (без потенциала) решена следующая обратная задача: каким образом по заданной плотности вещества этого поля (как функции масштабного фактора) можно найти его лагранжиан, давление, уравнение состояния, скорость звука и т. д.? На основе полученных формул, справедливых как в РТГ, так и в ОТО, довольно удобно анализировать модели $k$-эссенции вида (13). В частности, отсюда сразу получается простейшая объединенная модель темной энергии и темной материи в ОТО [8].

В третьем разделе на основе полученных общих формул для обратной задачи мы показываем, что модель $k$-эссенции с постоянным $\nu$, предложенная в работе [6], не может быть жизнеспособной из-за ее неустойчивости, приводящей к экспоненциальному разрушению фридмановской симметрии. Также строго доказывается, что для произвольного лагранжиана скалярного поля $k$-эссенции типа (13) в рамках РТГ невозможно удовлетворить одновременно следующим критериям: а) наличию космологического ускорения в настоящее время, б) гравитационной устойчивости 
такого скалярного поля относительно малых возмущений, в) наличию точки поворота фридмановского сценария на поздних временах для того, чтобы гарантировать соблюдение принципа причинности, и г) возможности достижения высоких плотностей на этапе сжатия (более $10^{3}$ Гэв), необходимых для существования впоследствии горячей фридмановской фазы. Отмечается, что в качестве возможной альтернативы всем этим критериям удовлетворяет скалярное поле экпирозиса [9]-[12].

В четвертом разделе показано, что модели $k$-эссенции рассматриваемого типа могут оказаться жизнеспособными в рамках плоского бесконечного фридмановского сценария ОТО. Приводятся конкретные модели соответствующих лагранжианов скалярных полей.

В заключении кратко перечислены основные результаты работы.

\section{2. ОБРАТНАЯ ЗАДАЧА В МОДЕЛИ $k$-ЭССЕНЦИИ БЕЗ ПОТЕНЦИАЛА}

Итак, рассмотрим модель скалярного поля (13), развивающегося на фоне однородной и изотропной (фридмановской) Вселенной. В этом случае скалярное поле зависит только от времени и, соответственно,

$$
X=\frac{1}{2} \dot{\phi}^{2}
$$

Обозначая производные как

$$
K_{X} \equiv \frac{d K}{d X}, \quad K_{X X} \equiv \frac{d K_{X}}{d X},
$$

запишем уравнения скалярного поля с лагранжианом (13), (15) в виде

$$
\left(K_{X}+2 X K_{X X}\right) \ddot{\phi}+3 \frac{\dot{a}}{a} K_{X} \dot{\phi}=0 .
$$

Если ввести функцию $G(X)$ :

$$
G(X)=\frac{1}{2 X}\left(1+2 \frac{X K_{X X}}{K_{X}}\right),
$$

и новую переменную $F$ такую, что

$$
G(X)=\frac{d}{d X} \ln F, \quad F=\exp \left(\int^{X} G(X) d X\right),
$$

то уравнение (16) сводится к уравнению

$$
\frac{\dot{F}}{F}+3 \frac{\dot{a}}{a}=0 .
$$

Отсюда следует, что

$$
F=\frac{\lambda}{a^{3}}, \quad \lambda=\text { const }>0 .
$$

Из равенств (17), (18) также легко установить соотношение

$$
\frac{K_{X X}}{K_{X}}=\frac{(F / \sqrt{X})_{X}}{F / \sqrt{X}},
$$


которое позволяет найти лагранжиан $K$ и его производную $K_{X}$ в виде

$$
\begin{gathered}
K_{X}=C \frac{F}{\sqrt{X}}, \quad C=\text { const }>0, \\
K=C \int \frac{F}{\sqrt{X}} d X=2 C \int F d \sqrt{X} .
\end{gathered}
$$

Первый интеграл (21) был получен в работе [13].

Найдем теперь выражения для плотности и давления, соответствующих лагранжиану (13). По определению записываем равенства

$$
T_{\mu \nu}=\frac{2}{\sqrt{-g}} \frac{\delta L}{\delta g^{\mu \nu}}=(p+\rho) u_{\mu} u_{\nu}-p g_{\mu \nu}, \quad u_{\mu}=\frac{\partial_{\mu} \phi}{2 \sqrt{X}}=(1, \overrightarrow{0}) .
$$

Из соотношений (13) и (23) имеем

$$
\begin{aligned}
& \rho=2 X K_{X}-K, \\
& p=K .
\end{aligned}
$$

Чтобы получить зависимость $\rho=\rho(a)$, следует найти обратную к функции (18) функцию $X=X(F)$ и подставить ее в формулы (24) и (25). На практике чаще необходимо решать обратную задачу: выбирая конкретный вид плотности $\rho=\rho(a)$, чтобы удовлетворить ряду физических требований (например, наличию космологического ускорения ( $\ddot{a}>0)$, заданному поведению при $a \rightarrow 0$ и при $a \rightarrow \infty$ и т. д.), нужно найти соответствующий лагранжиан $K(X)$. Вместо зависимости от масштабного фактора $а$ удобнее искать зависимость от переменной $F(19)$, считая, что задана функция $\rho(F)$. Подставляя в равенство $(24)$ соотношения $(21),(22)$ и интегрируя по частям, записываем выражение

$$
\rho=2 C \int^{F} \sqrt{X} d F .
$$

Поэтому

$$
X=\frac{1}{4 C^{2}}\left(\rho_{F}\right)^{2} .
$$

Если найти отсюда обратную зависимость $F=F(X)$, тогда можно получить плотность энергии скалярного поля как функцию $X: \rho=\rho(F(X))$. Аналогично вычисляется давление. После интегрирования по частям из формулы (22) находим

$$
p=K=2 C \int^{X} F d \sqrt{X}=F \rho_{F}-\rho .
$$

Уравнение состояния и выражение для скорости звука в такой среде принимают очень простой вид:

$$
\begin{gathered}
\omega=\frac{p}{\rho}=-1+\frac{F \rho_{F}}{\rho} \\
c_{\mathrm{S}}^{2} \equiv \frac{d p / d X}{d \rho / d X}=\frac{d p / d F}{d \rho / d F}=\frac{F \rho_{F F}}{\rho_{F}} .
\end{gathered}
$$


Скорость звука не может быть отрицательной, в противном случае модель неустойчива относительно малых возмущений плотности [14], [15].

Покажем теперь на основе общих формул (28)-(30), что модель $k$-эссенции типа (13) нельзя использовать в РТГ в качестве модели темной энергии, а в рамках ОТО она вполне жизнеспособна.

\section{3. МОДЕЛЬ $k$-ЭССЕНЦИИ В РТГ}

В первых работах по РТГ [4], [5], в которых была обоснована необходимость введения квинтэссенции, представляющей темную энергию, ее плотность была выбрана в простейшем степенном виде

$$
\rho(F)=F^{\nu}
$$

для того, чтобы она определялась только одной константой $\nu$ такой, что $\omega=-1+\nu$, а также для того, чтобы выполнялось условие, гарантирующее наличие точки поворота для больших значений масштабного фактора: $\rho(0)=0$. В нашей работе [6] была предложена конкретная модель лагранжиана скалярного поля $k$-эссенции типа (13), которая дает плотность (31). Используя формулы (27), (28) для обратной задачи, легко найти лагранжиан $K$ :

$$
\begin{gathered}
K=F \rho_{F}-\rho=(\nu-1) F^{\nu}, \\
X=\frac{1}{4 C^{2}}\left(\rho_{F}\right)^{2}=\frac{\nu^{2}}{4 C^{2}} F^{2(\nu-1)}, \quad F=\left(\frac{4 C^{2}}{\nu^{2}} X\right)^{1 / 2(\nu-1)}, \\
K=-\epsilon X^{\nu / 2(\nu-1)}, \quad \epsilon=(1-\nu)\left(\frac{4 C^{2}}{\nu^{2}}\right)^{\nu / 2(\nu-1)}, \quad \nu \neq 0,1,
\end{gathered}
$$

что совпадает с формулой (14).

K сожалению, модель (31) неустойчива, так как квадрат скорости звука для всех значений $F$ отрицателен:

$$
c_{\mathrm{s}}^{2}=\frac{F \rho_{F F}}{\rho_{F}}=\nu-1<-\frac{1}{3} .
$$

Ограничение сверху на параметр $\nu$ вида $\nu<2 / 3$ является следствием космологического ускорения в настоящее время.

Покажем, что и любой другой лагранжан типа (13) либо явно противоречит данным наблюдений, либо приводит к неустойчивостям.

Функция $\rho=\rho(F)$ должна быть монотонной, поэтому ее первая производная должна быть неотрицательной, $\rho_{F} \geqslant 0$. В силу условия $c_{\mathrm{s}}^{2} \geqslant 0$ из выражения (30) следует, что и вторая производная также должна быть неотрицательной, $\rho_{F F} \geqslant 0$. Таким образом, функция $\rho=\rho(F)$ должна быть растущей (с ростом $F$ ) функцией, не имеющей перегибов. Отсюда сразу следует, что в рамках ОТО ее нельзя использовать в качестве объединенной модели инфляции (при $a \rightarrow 0$ ) и темной энергии $($ при $a \rightarrow \infty)$, так как инфляционный разлет предполагает примерное постоянство плотности энергии скалярного поля с последующей неизбежной сменой знака $\rho_{F F}$.

Рассмотрим теперь, какие ограничения накладывает на функцию $\rho(F)$ положительность наблюдаемого значения космологического ускорения и необходимость разворота $(\dot{a}=0)$ при некотором минимальном значении $F_{\min }=\lambda / a_{\max }^{3}$, необходимое 
для того, чтобы не нарушался принцип причинности и существовало предельное значение масштабного фактора $a_{\max } \gg 1$.

Большим значениям масштабного фактора отвечают малые значения переменной $F$. Не ограничивая общности, положим $\lambda=1$, так что $F=a^{-3}$, и запишем фридмановские уравнения РТГ (6), (7) в виде

$$
\begin{gathered}
\frac{3}{8 \pi}\left(\frac{\dot{a}}{a}\right)^{2}=\rho_{\mathrm{M}}+\rho-\frac{m^{2}}{16 \pi}\left[1-\frac{3 F^{2 / 3}}{2 \beta^{4}}+\frac{F^{2}}{2}\right], \\
\frac{3}{4 \pi} \frac{\ddot{a}}{a}=-\left(\rho_{\mathrm{M}}+\rho+3 p\right)-\frac{m^{2}}{8 \pi}\left[1-F^{2}\right] .
\end{gathered}
$$

Плотность темной материи обозначим как

$$
\begin{gathered}
\rho_{\mathrm{M}}=\Omega_{\mathrm{M}} \rho_{\mathrm{c}} \frac{a_{0}^{3}}{a^{3}} \equiv A F, \\
\Omega_{\mathrm{M}} \cong 0.3, \quad \rho_{\mathrm{c}}=\frac{3}{8 \pi} H_{0}^{2}, \quad A=\text { const. }
\end{gathered}
$$

Здесь $\rho_{\mathrm{c}}-$ критическая плотность. Энергией космического микроволнового излучения в рассматриваемой области $a>a_{0}$, где $a_{0}$ - современное значение масштабного фактора, можно пренебречь [16], также как и членом, пропорциональным $F^{2 / 3}$, поскольку $\beta^{4} \geqslant a_{\max }^{4}$.

В настоящую эпоху $\left(a=a_{0}, F=F_{0}=a_{0}^{-3}, \rho_{F}^{0}=\rho_{F}\left(F_{0}\right)\right)$ ускорение положительно $(\ddot{a}>0)$, поэтому из формул $(28),(34),(35)$ находим ограничение

$$
A F_{0}+3 F_{0} \rho_{F}^{0}-2 \rho_{0}<-\frac{m^{2}}{8 \pi}\left[1-F_{0}^{2}\right]
$$

Это соотношение выполняется независимо от величины $a_{0}$.

Наконец, условие существования точки разворота $\left(a=a_{\max }\right)$ в силу уравнения (33) означает, что для некоторого $F_{\min }=a_{\max }^{-3} \ll 1$ справедливо равенство

$$
\rho_{\min }+A F_{\min }=\frac{m^{2}}{16 \pi}\left[1+\frac{F_{\min }^{2}}{2}\right] .
$$

Как оценить $\rho_{\min }$, если известны $\rho_{0}$ и $\rho_{F}^{0}$ ? Обозначим через $\tilde{\rho}(F)$ касательную к кривой плотности $\rho(F)$ в точке $F_{0}$. Эта прямая пересечет вертикальную ось в точке $\tilde{\rho}_{\text {min }}$. По определению касательной

$$
\tilde{\rho}(F)=\rho_{0}+\left(F-F_{0}\right) \rho_{F}^{0} .
$$

Поэтому

$$
\tilde{\rho}_{\min }=\tilde{\rho}(0)=\rho_{0}-F_{0} \rho_{F}^{0} .
$$

Космологическое ускорение в настоящую эпоху обеспечивается членами в уравнении (34), отвечающими скалярному полю. Тогда, как следует из формул (28) и (34), $3 F_{0} \rho_{F}^{0}-2 \rho_{0}<0$. Таким образом, $\tilde{\rho}_{\min }>\rho_{0} / 3>0$.

Так как на касательной $\tilde{\rho}_{F F}=0$, то левее точки $F_{0}$ кривая $\rho(F)$ должна лежать выше нее, в противном случае нарушено условие $\rho_{F F} \geqslant 0$. Поэтому минимум плотности $\rho_{\min }=\rho(0)$ также должен быть строго положительным:

$$
\rho_{\min }>\tilde{\rho}_{\min }>0 \text {. }
$$


Это еще раз подтверждает неприемлемость в РТГ модели темной энергии (14), для которой $\rho_{\min }=0$.

Из уравнений (37), (39) и условия (40) можно получить неравенство

$$
\rho_{0}-F_{0} \rho_{F}^{0}+A F_{\min } \leqslant \frac{m^{2}}{16 \pi}\left[1+\frac{F_{\min }^{2}}{2}\right] .
$$

Умножая его на два и складывая с (36), получаем неравенство

$$
F_{0} \rho_{F}^{0}+A\left(F_{0}+2 F_{\text {min }}\right) \leqslant \frac{m^{2}}{8 \pi}\left[F_{0}^{2}+\frac{F_{\text {min }}^{2}}{2}\right] .
$$

Так как все слагаемые в левой части положительны, то

$$
A F_{0} \leqslant \frac{m^{2}}{8 \pi}\left[F_{0}^{2}+\frac{F_{\mathrm{min}}^{2}}{2}\right] .
$$

Отсюда ввиду того, что $F_{\min }<F_{0}$, вытекает неравенство

$$
\frac{m^{2}}{8 \pi} \geqslant \frac{A F_{0}}{F_{0}^{2}+F_{\min }^{2} / 2} \geqslant \frac{2 A F_{0}}{3 F_{0}^{2}}=\frac{2}{3} \frac{0.3 \rho_{\mathrm{c}}}{F_{0}^{2}},
$$

которое дает сильную оценку снизу на массу гравитона вида $m^{2} / 16 \pi a_{0}^{6} \geqslant 0.1 \rho_{\mathrm{c}}$. Рассмотрим теперь этап сжатия, точнее тот его участок, где масштабный фактор уменьшается от значения $a_{0}$. Так как отрицательный член $-m^{2} / 16 \pi a^{6}$ в формуле для плотности (6) на стадии сжатия растет быстрее всего при уменьшении $a$, то он “догонит" слагаемое с темной материей (пропорциональное $a^{-3}$ ) практически сразу, т. е. в этой точке разворота $a_{\text {min }}$ будет иметь такой же порядок, что и $a_{0}$. Поэтому никакой горячей стадии в космологическом сценарии с такой моделью темной энергии быть не может. Это справедливо для любой функции $\rho(F)$, удовлетворяющей вышеперечисленным критериям, что и требовалось доказать.

Таким образом в РТГ возникает необходимость выбрать другую самомогласованную модель квинтэссенции, которая обеспечивала бы космологическое ускорение в настоящую эпоху, а в будущем приводила бы к смене режима расширения $(\dot{a}>0)$ на режим сжатия $(\dot{a}<0)$. Этот последний режим не является зеркальным отражением первого, так как падающие и растущие моды возмущений не являются одинаковыми [17]. Поэтому, кстати, фридмановское решение в РТГ со скалярным полем (14) нестабильно не только на этапе ускорения стадии расширения, но и на этапе сжатия.

Как показал Стейнхардт с соавторами [11], анизотропизации, разрушающей фридмановский сценарий, в режиме сжатия можно избежать, если уравнение состояния лидирующей компоненты вещества удовлетворяет неравенству $\omega>1$. Такую среду создает скалярное поле $\phi=\phi(\tau)$, представленное обычным лагранжианом

$$
L_{\phi}=\sqrt{-g}\left(\frac{1}{2} g^{\mu \nu} \nabla_{\mu} \phi \nabla_{\nu} \phi-V(\phi)\right),
$$

но с отрицательным (для $\phi<0)$ потенциалом [9] (так называемый сценарий с экпирозисом)

$$
V(\phi)=V_{0}\left(1-e^{-c \phi}\right) F(\phi)
$$


где $V_{0}, c=$ const $>0, F(\phi)$ - положительная функция, обеспечивающая выполнение условия $V(\phi) \rightarrow 0$ при $\phi \rightarrow-\infty$ и равная $F(\phi) \cong 1$ правее минимума потенциала. Роль гравитационной постоянной, обеспечивающей сегодняшнее ускорение, в данном случае играет величина $V_{0}-m^{2} / 32 \pi>0$. Подробный анализ сценария с экпирозисом в РТГ выходит за рамки настоящей работы и будет рассмотрен отдельно.

\section{4. МОДЕЛЬ $k$-ЭССЕНЦИИ В ОТО}

В рамках ОТО модели с лагранжианами типа (13) вполне жизнеспособны, но, как было показано выше, важно то, что значение $\rho(0)$ должно быть больше нуля, так как только в этом случае соблюдается условие устойчивости $c_{\mathrm{s}}^{2}>0$. Именно величина $\rho(0)$ и играет роль космологической постоянной (темной энергии), приводящей к ускорению. Линейный член (напомним, что здесь рассматривается область больших $a>a_{0}$, т. е. малых $F$ ) при разложении в нуле описывает темную материю [8], [13]:

$$
\rho(F)=\rho(0)+\rho_{F}(0) F
$$

В качестве простейшего примера такой модели можно выбрать следующую:

$$
\rho(F)=A+B F+C F^{2} .
$$

Тогда согласно выражению (27) находим, что

$$
X=\frac{(B+2 C F)^{2}}{4 C^{2}}, \quad F=\sqrt{X}-\frac{B}{2 C} .
$$

Как и следовало ожидать, линейной слагаемое, отвечающее темной материи, не дает вклада в давление (28):

$$
p(F)=C F^{2}-A,
$$

а постоянный член, соответствующий темной энергии, - в скорость звука (30):

$$
c_{\mathrm{s}}^{2}=\frac{1}{1+B / 2 C F}
$$

Так как параметр $B$ определяет плотность темной материи, то $B>0$. Поэтому из условия $c_{\mathrm{s}}^{2}>0$ следует, что и $C>0$. Тогда для диапазона $0<F<\infty$ квадрат скорости звука принимает значения в положительном интервале $0<c_{\mathrm{s}}^{2}<1$.

Найдем также уравнение состояния (29) и его асимптотику при малых $F$ :

$$
\omega=\frac{p}{\rho}=-\frac{A-C F^{2}}{A+B F+C F^{2}} \cong-1+\frac{B}{A} F+\left[\frac{2 C}{A}-\left(\frac{B}{A}\right)^{2}\right] F^{2} .
$$

Лагранжиан этой модели получаем в виде (13) (не снижая общности отождествляя константы $C$ в $(21)$ и (44))

$$
K=p=C X-B \sqrt{X}-A+\frac{B^{2}}{4 C},
$$

впервые рассмотренном в работе [13]. 
Обратная задача позволяет работать с полями, лагранжиан которых невозможно выписать явно как функцию этих полей. Таким примером является обобщенная модель газа Чаплыгина. На основании общих соотношений обратной задачи (28)-(30) формулы для давления (лагранжиана) и скорости звука в этой модели, задаваемой плотностью [8]

$$
\rho(F)=\left(A+B F^{1+\alpha}\right)^{1 /(1+\alpha)}, \quad A, B>0,
$$

получаются тривиальным образом:

$$
p=-\frac{A}{\rho^{\alpha}}, \quad c_{\mathrm{s}}^{2}=\frac{1}{1+(B / 2 A) F^{1+\alpha}} .
$$

Из равенства (27) следует, что при $\alpha \neq 1$ явную зависимость $F=F(X)$ и, соответственно, лагранжиан как функцию $X$ найти невозможно:

$$
X=\frac{\left(B F^{\alpha}\right)^{2}}{4 C^{2}\left(A+B F^{1+\alpha}\right)^{2 \alpha /(1+\alpha)}} .
$$

В простом частном случае собственно газа Чаплыгина $(\alpha=1)$ лагранжиан выписывается явно:

$$
F(X)=\frac{2 C \sqrt{A X}}{\sqrt{B\left(B-4 C^{2} X\right)}}, \quad K(X)=-\sqrt{\frac{A}{B}\left(B-4 C^{2} X\right)} .
$$

Очевидно, что скалярное поле должно подчиняться неравенству $\dot{\phi}^{2} \leqslant A B / 2 C^{2}$.

Возможна и другая постановка задачи. Пусть задано уравнение состояния среды $p=p(\rho) \equiv f(\rho), d f / d \rho>0$, которую нужно моделировать скалярным полем $k$-эссенции. В этом случае из закона сохранения вещества (8) находим зависимость $F=F(\rho)$ :

$$
F=b \exp \left(\int^{\rho} \frac{d \rho}{\rho+f(\rho)}\right)
$$

где $b>0$ - постоянная интегрирования. Тогда из равенства (27) можно получить связь $X=X(\rho)$ :

$$
X=\left(\frac{\rho+f(\rho)}{2 C F}\right)^{2}=\left(\frac{\rho+f(\rho)}{2 C b \exp \left(\int^{\rho} \frac{d \rho}{\rho+f(\rho)}\right)}\right)^{2} .
$$

Если существует явная обратная функция $\rho=\rho(X)$, то тогда нетрудно найти лагранжиан (давление) как функцию $X$. Для обобщенного газа Чаплыгина из формулы (52) получаем, что

$$
F=b \exp \left(\frac{1}{1+\alpha} \ln \left(\rho^{1+\alpha}-A\right)\right)
$$

откуда

$$
\rho(F)=\left(A+B F^{1+\alpha}\right)^{1 /(1+\alpha)}, \quad B=b^{-(1+\alpha)},
$$

что совпадает с выражением (49). Уравнение (53) в этом случае приводит к равенству

$$
X=\left(\frac{\left(\rho^{1+\alpha}-A\right)^{\alpha /(1+\alpha)}}{2 C b \rho^{\alpha}}\right)^{2}
$$


и, вообще говоря, не позволяет найти $\rho(X)$ в явном виде, за исключением случая $\alpha=1$, когда

$$
L_{\phi}=-\frac{A}{\rho}=-\sqrt{\frac{A}{B}\left(B-4 C^{2} X\right)},
$$

что также совпадает с (51).

\section{5. ЗАКЛЮЧЕНИЕ}

В настоящей работе рассмотрено скалярное поле квинтэссенции с нетривиальной кинетической частью без потенциала ( $k$-эссенции), развивающейся в плоской фридмановской Вселенной. Решена обратная задача построения Лагранжиана такого скалярного поля исходя из зависимости его плотности энергии от масштабного фактора $\rho=\rho(a)$. Моделирование темной энергии таким скалярным полем в РТГ обеспечивает современное значение космологического ускорения и наличие точки поворота на поздних временах. Показано, что в этой теории любое скалярное поле указанного типа является патологическим либо в силу гравитационной неустойчивости этого решения относительно возмущений с длинами волн, находящимися далеко за космологическим горизонтом, т. е. зависящими только от времени, либо в силу недопустимо быстрого окончания стадии сжатия. В качестве непротиворечивой модели квинтэссенции в РТГ предложена модель Стейнхардта-Турока для скалярного поля с экпирозисом, которая используется в ОТО для построения различных осциллирующих космологических сценариев. Отмечается, что скалярное поле $k$-эссенции в рамках бесконечного плоского фридмановского решения ОТО жизнеспособно и, более того, может представлять собой модель не только темной энергии, но и темной материи.

\section{Список литературы}

[1] А.А. Логунов, М.А. Мествиришвили, Ю.В. Чугреев, Масса гравитона и развитие фридмановской Вселенной, Изд-во МГУ, М., 1987; ТМФ, 74 (1987), 3-15.

[2] Ю.В. Чугреев, "Принцип Маха для космологических решений в релятивистской теории гравитации", Писъма в ЭЧАЯ, 12:2(193) (2015), 281-298.

[3] М. А. Мествиришвили, Ю. В. Чугреев, "Фридмановская модель эволюции Вселенной в релятивистской теории гравитации”, ТМФ, 80:2 (1989), 305-312.

[4] С. С. Герштейн, А. А. Логунов, М. А. Мествиришвили, Н. П. Ткаченко, "Масса гравитона, квинтэссенция и осциллирующий характер эволюции Вселенной”, ЯФ, 67:8 (2004), $1618-1626$.

[5] С. С. Герштейн, А. А. Логунов, М.А. Мествиришвили, "Космологическая постоянная и пространство Минковского", ЭЧАЯ, 38:3 (2007), 569-586.

[6] М. А. Мествиришвили, К. А. Модестов, Ю. В. Чугреев, "Скалярное поле квинэссенции в релятивистской теории гравитации", ТМФ, 152 (2007), 551-560, arXiv: gr-qc/0612105.

[7] Ю.В. Чугреев, “Темная энергия и масса гравитона в Ближней Вселенной”, Писъма в ЭЧАЯ, 13:1(199) (2016), 66-79.

[8] R. J. Sherrer, "Purely kinetic $k$-essence as unified dark matter", Phys. Rev. Lett., 93:1 (2004), 011301, 4 pp., arXiv: astro-ph/0402316.

[9] P. J. Steinhardt, N. Turok, "A cyclic model of the Universe", Science, 296:5572 (2002), 1436-1439, arXiv: hep-th/0111030.

[10] P. J. Steinhardt, N. Turok, "Cosmic evolution in a cyclic universe", Phys. Rev. D, 65:12 (2002), 126003, 20 pp., arXiv: hep-th/0111098. 
[11] J. K. Erickson, D. H. Wesley, P. J. Steinhardt, N. Turok, "Kasner and mixmaster behavior in universes with equation of state $\omega \geqslant 1$ ", Phys. Rev. D, 69:6 (2004), 063514, 11 pp., arXiv: hep-th/0312009.

[12] A. Ijjas, P. J. Steinhardt, "Classically stable nonsingular cosmological bounces", Phys. Rev. Lett., 117:12 (2016), 121304, 5 pp., arXiv: 1606.08880.

[13] L.P. Chimento, "Extended tachyon field, Chaplygin gas and solvable $k$-essence cosmologies", Phys. Rev. D, 69:12 (2004), 123517, 10 pp., arXiv: astro-ph/0311613.

[14] J. Garriaga, V. F. Mukhanov, "Perturbations in k-inflation", Phys. Lett. B, 458:2-3, 1999, arXiv: hep-th/9904176.

[15] V. Mukhanov, Physical Foundations of Cosmology, Cambridge Univ. Press, Cambridge, 2012.

[16] Д. С. Горбунов, В. А. Рубаков, Введение в теорию ранней Вселенной. Теория горячего большого взрыва, Ленанд, М., 2016.

[17] Д. С. Горбунов, В. А. Рубаков, Введение в теорию ранней Вселенной. Космологические возмущения. Инфляционнал теория, Красанд, М., 2016.

Поступила в редакцию 26.01.2017

После доработки 7.04.2017 